Rechtsmedizin 2021 · 31:405-407 https://doi.org/10.1007/s00194-021-00511-7 Angenommen: 12. Mai 2021 Online publiziert: 12 . Juli 2021

(c) Der/die Autor(en) 2021

\section{Konsensus-Statement von 7 Fachgesellschaften* und der Medizinischen Kinderschutzhotline zur Diagnostizierbarkeit des Schütteltraumas}

\author{
Deutsche Gesellschaft für Kinderschutz in der Medizin \\ (DGKiM), Deutsche Gesellschaft für Rechtsmedizin (DGRM), \\ Deutsche Gesellschaft für Kinder- und Jugendmedizin \\ (DGKJ), Deutsche Gesellschaft für Sozialpädiatrie und \\ Jugendmedizin (DGSPJ), Gesellschaft für Neuropädiatrie \\ (GNP), Deutsche Gesellschaft für Kinder- und \\ Jugendpsychiatrie, Psychosomatik und Psychotherapie \\ (DGKJP), Gesellschaft für Pädiatrische Radiologie (GPR), und \\ Medizinische Kinderschutzhotline ${ }^{+}$
}

\section{B. Herrmann ${ }^{1} \cdot$ R. B. Dettmeyer ${ }^{2} \cdot$ S. Banaschak ${ }^{3}$}

${ }^{1}$ Klinik für Neonatologie und allgemeine Pädiatrie, Klinikum Kassel GmbH, Kassel, Deutschland; ${ }^{2}$ Institut für Rechtsmedizin, Universitätsklinikum Gießen, Gießen, Deutschland; ${ }^{3}$ Institut für Rechtsmedizin, Universität zu Köln, Medizinische Fakultät und Uniklinik Köln, Köln, Deutschland
+ Bei der Erstellung des Beitrages „Die Medizinische Kinderschutzhotline: Beratung für Angehörige der Heilberufe bei Kinderschutzfragen" in pädiatrische praxis 2021, Band 95/3 war den Autoren nicht bewusst, in welchem Kontext der Artikel abgedruckt wird. Den Autoren ist es wichtig, sich von den im selben Band abgedruckten Artikeln zu distanzieren und gemeinsam einen seriösen fachlichen Konsens zu publizieren.

* Die Vertreter der einzelnen Fachgesellschaften werden am Beitragsende in einer Box genannt.

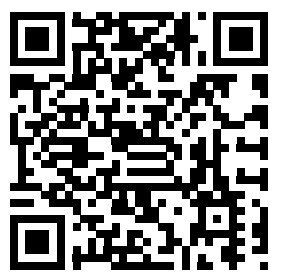

QR-Code scannen \& Beitrag online lesen
Eine Reihe von Autoren hat in der Zeitschrift pädiatrische praxis, Ausgabe 95/2021, eine Serie von Artikeln zum Schütteltraumasyndrom (STS) bzw. zu misshandlungsbedingten Kopfverletzungen publiziert, die in der Summe die Diagnostizierbarkeit des Schütteltraumas anzweifeln [13-15].

Dieses Konsensus-Statement der oben genannten Fachgesellschaften bezieht sich daher auf die grundsätzliche Frage der Diagnostizierbarkeit des Schütteltraumas. Weitere, insbesondere auch schwerwiegende fachlich-methodische Kritikpunkte an den Arbeiten werden hier ausdrücklich nicht angesprochen ${ }^{1}$.

1 Sollte eine Leserin/ein Leser Bedarf an weiteren Hinweisen dazu haben, kann er sich an die genannten Autoren des Konsensus-Statements wenden.
Aus Sicht der oben genannten Fachgesellschaften ist grundsätzlich zwischen einer medizinisch-wissenschaftlichen Diskussion und einer Sachverständigentätigkeit bei Gericht zu unterscheiden. In einer fachlichen Diskussion können auch schwache Hypothesen und gelegentlich auch Behauptungen aufgestellt werden. Für derartige unbelegte Äußerungen ist der Strafprozess jedoch nicht der geeignete Ort. Dort werden fachliche Schlussfolgerungen auf wissenschaftlicher Basis vorgetragen und sollen dem Gericht helfen, eine durch medizinische Fakten gestützte Entscheidung zu treffen (z. B. [17]).

Die Artikel der verschiedenen Autoren(gruppen) nehmen bei ihren Ausführungen anscheinend auf einen (gemeinsam?) als Gutachter der Verteidigung bearbeiteten Fall Bezug. Ein derartiger Zusammenhang entspräche einem nichtdekla- 
rierten Interessenkonflikt. Ein solcher wird aber nicht offengelegt.

Die Diagnose des Schütteltraumas wird entweder in einem klinischen oder bei verstorbenen Kindern - rechtsmedizinischen Zusammenhang gestellt. Hierzu gibt es etablierte Untersuchungsabläufe, die auch in deutschsprachigen Leitlinien festgehalten sind $[9,10]$. Des Weiteren gibt es aktuelle Publikationen - auch gemeinsam von Pädiatern und Rechtsmedizinern -, die sich mit der Diagnose des Schütteltraumas befassen (z. B. [2, 3, 6, $7,16])$, internationale Leitlinien und Konsensuspapiere [1,5] und systematische Reviews [12].

Häufig steht am Beginn der Diagnostik eine (neurologische) Auffälligkeit des Kindes, die dann zu weiteren Untersuchungsschritten führt (u.a. mit der Klärung bekannter Differenzialdiagnosen). Auf diese klinische bzw. postmortale Diagnostik soll in diesem Statement nicht detailliert eingegangen werden. Wir verweisen dazu auf die Literatur (einige Arbeiten s. Literaturverzeichnis) und entsprechende Lehrbücher (z. B. [8, 11]).

Es erfolgt nach international einvernehmlichem fachlichem Standard keine „automatische“ Diagnose eines Schütteltraumas anhand der von den Autoren mehrfach zitierten "diagnostischen Trias", wenn ein Subduralhämatom in Kombination mit retinalen Blutungen und einer Enzephalopathie festgestellt wird. Die Diagnose beruht auf anamnestischen, klinischen, radiologischen und ophthalmologischen Befunden, unter Berücksichtigung aller relevanten differenzialdiagnostischen Erwägungen. Diese gehören ebenso zur Erörterung jedes Einzelfalls wie weiterführende Untersuchungen, u. a. des Skelettsystems (Röntgenskelettscreening) zur Prüfung evtl. weiterer bestehender Verletzungen. Am Ende des diagnostischen Prozesses wird nach Empfehlung der durch die Arbeitsgemeinschaft der Wissenschaftlichen Medizinischen Fachgesellschaften (AWMF) koordinierten S3+-Kinderschutzleitlinie [9] in einem multiprofessionellen Abwägungsprozess die Diagnose gestellt, und zwar unter Berücksichtigung aller diagnostischen Ergebnisse und der zu diesem Zeitpunkt bekannten Anamnese. Werden neue/andere Anknüpfungstatsachen bekannt (z.B. Berichte über ein
Unfallereignis), so muss neu beurteilt werden.

Aber die Diagnose kann - auch postmortal - unter Berücksichtigung der oben genannten Standards verlässlich und sicher gestellt werden. Die von von Voss (2021; [14]) behauptete Kontroverse besteht nicht. Es handelt sich um eine Pseudokontroverse, die teils von schon bekannten Protagonisten (vorwiegend aus den USA), teils von anderen Autoren (das bekannteste Beispiel waren Autoren aus Schweden; [4]) immer wieder versucht wird. Bislang ist keine substanzielle Widerlegung des Konzeptes des Schütteltraumas und des damit verbundenen Pathomechanismus und seinen Folgen gelungen (zusammenfassend [3, 8], zur schwedischen Publikation [4]).

Die von den Autoren Wiederer et al. (2021, [15]) bereits im Titel („Schütteltrauma vs. SIDS ...") gegeneinander gestellten Entitäten Schütteltrauma und plötzlicher Kindstod (,sudden infant death syndrome", SIDS) haben nichts miteinander zu tun. Wenn ein Säugling retinale Blutungen und/oder ein Subduralhämatom aufweist, kann es sich definitionsgemäß nicht um einen plötzlichen Kindstod handeln. Dieser beruht ja u.a. gerade darauf, dass es keine (!) derartigen oder anderweitige Befunde gibt. Die Frage "Schütteltrauma vs. SIDS" können die Autoren nur aufwerfen, da sie eine Entstehung von Subduralhämatomen und retinalen Blutungen allein durch eine Hypoxie unterstellen. Diese Unterstellung ist schon längst widerlegt. Daher kann bei diesen Befunden kein plötzlicher Kindstod vorliegen.

\section{Umgang mit der gestellten Diagnose}

Ist ein Kind lebensbedrohlich verletzt, so kommt - unabhängig von der bestehenden Schweigepflicht - eine Meldung an die Polizei und die Staatsanwaltschaft oder auch nach $\S 4$ des Gesetzes zur Kooperation und Information im Kinderschutz (KKG) an das Jugendamt in Betracht. Üblicherweise werden derartige Entscheidungen in einer Kinderschutzgruppe getroffen, in der alle beteiligten Berufsgruppen und medizinische Fachrichtungen vertrauensvoll zusammenarbeiten. Diese Zusammenarbeit hat sich in den zurücklie- genden Jahren intensiv entwickelt und wird entsprechend den Empfehlungen der Deutschen Gesellschaft für Kinderschutz in der Medizin (DGKiM) und der Deutschen Akademie für Kinder- und Jugendmedizin (DAKJ) in mittlerweile über 180 Kinderschutzgruppen bundesweit praktiziert. Dabei gibt es aufgrund der unterschiedlichen Tätigkeiten verschiedene Herangehensweisen, aber keinen fachlichen Dissens zwischen z. B. Pädiatrie und Rechtsmedizin.

Es ist uns als wissenschaftlichen Fachgesellschaften wichtig zu betonen, dass eine fundierte (Differenzial-)Diagnostik in allen Verdachtsfällen von Kindesmisshandlung die Basis einer weitergehenden, auch rechtlichen Beurteilung ist. Die Eltern und ihre Kinder haben einen Anspruch darauf, dass nicht persönliche Interessen und wissenschaftlich unseriöse Diskussionen die medizinische Beurteilung prägen. Falschpositive und falsch-negative Diagnosestellungen haben in diesem Themenfeld dramatische Konsequenzen. Es ist unsere gemeinsame Verantwortung, für eine angemessene Beurteilung von Verdachtsfällen einzustehen - jeder mit seiner Fachkompetenz und in einem wissenschaftlich fundierten Abwägungsprozess.

\section{Korrespondenzadresse}

Prof. Dr. S. Banaschak

Institut für Rechtsmedizin, Universität zu Köln, Medizinische Fakultät und Uniklinik Köln Melatengürtel 60/62, 50823 Köln, Deutschland sibylle.banaschak@uk-koeln.de

Funding. Open Access funding enabled and organized by Projekt DEAL.

\section{Einhaltung ethischer Richtlinien}

Interessenkonflikt. B. Herrmann, R.B. Dettmeyer und S. Banaschak geben an, dass kein Interessenkonflikt besteht.

Für diesen Beitrag wurden von den Autoren keine Studien an Menschen oder Tieren durchgeführt. Für die aufgeführten Studien gelten die jeweils dort angegebenen ethischen Richtlinien.

Open Access. Dieser Artikel wird unter der Creative Commons Namensnennung 4.0 International Lizenz veröffentlicht, welche die Nutzung, Vervielfältigung, Bearbeitung, Verbreitung und Wiedergabe in jeglichem Medium und Format erlaubt, sofern Sie den/die ursprünglichen Autor(en) und die Quelle ordnungsgemäß nennen, einen Link zur Creative Commons Lizenz 
Infobox 1

Beteiligte Fachgesellschaften und ihre Vertreter

Deutsche Gesellschaft Kinderschutz in der Medizin (DGKiM), Dr. B. Herrmann

(Vorsitzender), Klinikum Kassel, Kassel Deutsche Gesellschaft für Rechtsmedizin (DGRM), Prof. S. Ritz-Timme (Präsidentin), Universitätsklinikum Düsseldorf

Deutsche Gesellschaft für Kinder- und Jugendmedizin (DGKJ), Prof. I. KrägelohMann, (Vizepräsidentin), Universitätsklinik Tübingen

Deutsche Gesellschaft für Sozialpädiatrie und Jugendmedizin (DGSPJ), Prof. U. Thyen (Präsidentin), Universitätsklinikum SchleswigHolstein, Lübeck

Gesellschaft für Neuropädiatrie (GNP), Prof. Dr. U. Schara (Präsidentin), Universitätsklinikum Essen, und Prof. Dr. M. Kieslich (Vizepräsident), Universitätsklinikum Frankfurt/Main

Deutsche Gesellschaft für Kinder- und Jugendpsychiatrie, Psychosomatik und Psychotherapie (DGKJP), Prof. Dr. M. Kölch (Präsident), Universitätsmedizin Rostock Gesellschaft für Pädiatrische Radiologie (GPR), Prof. Dr. H.-J. Mentzel (Präsident), Universitätsklinikum Jena und Medizinische Kinderschutzhotline Prof. J. Fegert, Universitätsklinikum Ulm

beifügen und angeben, ob Änderungen vorgenommen wurden.

Die in diesem Artikel enthaltenen Bilder und sonstiges Drittmaterial unterliegen ebenfalls der genannten Creative Commons Lizenz, sofern sich aus der Abbildungslegende nichts anderes ergibt. Sofern das betreffende Material nicht unter der genannten Creative Commons Lizenz steht und die betreffende Handlung nicht nach gesetzlichen Vorschriften erlaubt ist, ist für die oben aufgeführten Weiterverwendungen des Materials die Einwilligung des jeweiligen Rechteinhabers einzuholen.

Weitere Details zur Lizenz entnehmen Sie bitte der Lizenzinformation auf http://creativecommons.org/ licenses/by/4.0/deed.de.

\section{Literatur}

1. American Academy of Pediatrics, Narang SK, Fingarson A, Lukefahr J, Council on Child Abuse and Neglect (2020) Abusive head trauma in infants and children. Pediatrics 145:e20200203 (https:// pediatrics.aappublications.org/content/145/4/ e20200203. Zuletzt abgerufen am 28.03.2021)

2. Baz Bartels M, Banaschak S, Herrmann B (2019) Update Schütteltraumasyndrom. Monatsschr Kinderheilkd 167:891-899

3. Berthold O, Fegert JM (2019) Schütteltraumasyndrom - diagnostische Sicherheit trotz andauernder medialer Kontroverse. Monatsschr Kinderheilkd 167:426-433

4. Bilo RAC, Banaschak S, Herrmann B, Karst WA, Kubat B, Nijs HGT, van Rijn RR, Sperhake J, Stray-Pedersen A (2017) Using the table in the
Swedish review on shaken baby syndrome will not help courts deliver justice. Acta Paediatr 106:1043-1045

5. Choudhary A, Servaes S, Slovis TL, Palusci V, Hedlund G, Narang SK, Moreno JA, Dias MS, Christian CW, Nelson MD, Silvera VM, Palasis S, Raissaki M, Rossi A, Offiah A (2018) Consensus statement on abusive head trauma in infants and young children. Pediatr Radiol 48:1048-1065

6. Greeley CS (2015) Abusive head trauma: a review of the evidence base. Am J Radiol 204:967-973

7. Herrmann B (2016) Epidemiologie, Klinik und Konzept des Schütteltrauma-Syndroms. Pädiatr Prax 86:297-313

8. Herrmann B, Dettmeyer R, Banaschak S, Thyen U (Hrsg) (2016) Kindesmisshandlung. Medizinische Diagnostik, Intervention und rechtliche Grundlagen, 3. Aufl. Springer, Berlin Heidelberg

9. Kinderschutzleitlinienbüro (2019) AWMF S3+ Leitlinie Kindesmisshandlung, -missbrauch, -vernachlässigung unter Einbindung der Jugendhilfe und Pädagogik (Kinderschutzleitlinie), Langfassung 1.0, Februar 2019, AWMF-Registernummer: 027 - 069, S 238-250. https://www.awmf.org/ leitlinien/detail/II/027-069.html. Zugegriffen: 26 März2021

10. (2017) Leitlinie Die rechtsmedizinische Leichenöffnung. Langfassung 31.10.2017, AWMFRegisternummer: 054 - 001. https://www.awmf. org/leitlinien/detail/II/054-001.html.Zugegriffen: 26. März 2021

11. Madea B (Hrsg) (2015) Rechtsmedizin. Befunderhebung, Rekonstruktion, Begutachtung. Springer Berlin Heidelberg

12. Royal College of Paediatrics and Child Health (RCPCH) (2019) Child Protection Evidence-Systematic review on Head and Spinal Injuries. https://www.rcpch.ac.uk/resources/ child-protection-evidence-head-and-spinalinjuries. Zugegriffen: 28. März 2021

13. Schulz-Schaeffer WJ (2021) Misshandlungsbedingte Enzephalopathien: neuere Erkenntnisse ändern die Symptom-Trias. Pädiatr Prax 95:391-397

14. von Voss H (2021) Schütteltrauma-Verdacht: Herausforderungen an Gutachter und Gericht. Pädiatr Prax 95:373-378

15. Wiederer L, Ballweg A, Friederichs E, Eber $S$ (2021) Schütteltrauma vs. SIDS im Säuglingsalter: Reifungsverzögerung des autonomen Nervensystems als Differenzialdiagnose. Pädiatr Prax 95:379-390

16. Zinka B, Banaschak S, Mützel E (2018) Nachweissicherheit des Schütteltraumas. Bewertung von klassischer Symptomentrias, luzidem Intervall und Differenzialdiagnosen. Rechtsmedizin 28:474-481

17. Zinka B, Banaschak S, Mützel E (2019) Zur klassischen Symptomtrias bei Schütteltrauma. Rechtsmedizin 29:218

\section{Klaus-Steffen Saternus \\ Rechtsmediziner wollte ich nie werden}

Berlin: Lehmanns Media 2021, 1. Aufl., 341 S., (ISBN: 978-3-96543208-6), 24,95 EUR

Überraschend beginnt diese Biographie mit der 1944 noch üblichen Redewendung: „Ein Junge weint nicht!". Als vierjähriger Knabe stößt sich K den Fuß schmerzhaft an seinem Dreirad und hat den Impuls, den Schmerz - auch weinend - mitzuteilen: Der Beginn einer spannenden Autobiografie des Autors Klaus-Steffen Saternus, im Buch „K" genannt. Das Buch zeichnet seinen Lebensweg von Neustettin, die Flucht über die innerdeutsche Grenze in Berlin nach Köln, das Abitur und das MedizinStudium in Köln. Es folgen die Jahre der Assistenzarzt-Zeit: Unfallchirurgie, Pathologie, Humangenetik und - schließlich Rechtsmedizin, dort bis zur Habilitation, zunächst als auszubildender Mediziner, dann aber als Doktor und Professor - in Köln, Berlin und schließlich als Institutsdirektor in Göttingen.

Es ist ein Buch, das die politischen Verhältnisse in der DDR und in der BRD kritisch betrachtet und gleichzeitig das Fach „Rechtsmedizin“ mit K's umfangreichen Kenntnissen und Erfahrungen vorstellt. Mit einer sehr detaillierten Erinnerungsfähigkeit und einer durchgehend spannenden Schilderung von privaten Erlebnissen und forensischen Einzelfällen wird das wissenschaftliche Leben von $\mathrm{K}$ verdeutlicht. Die Biografie ist in ausgesprochen lockerer Sprache anschaulich und mit vielen biographischen Details geschrieben. Diese enthalten kritische Anmerkungen über Lebensumstände, Politik, Personen, Wissenschaft und das Fach Rechtsmedizin. Die Übergänge vom persönlichen Schicksal zu eher fachspezifischen Schilderungen sind fallbezogen und unschwer nachvollziehbar. Ein Schwerpunkt ist die eigene Forschung und deren Ergebnisse zum „Plötzlichen Kindstod“. Das Buch ist sowohl für Laien als auch - und besonders - für junge Rechtsmediziner empfehlenswert.

M. Oehmichen, Lübeck 